\title{
Automated Termination Analysis for Programs with Second-Order Recursion
}

\author{
Markus Aderhold \\ Technische Universität Darmstadt, Germany \\ aderhold@informatik.tu-darmstadt.de
}

\begin{abstract}
Many algorithms on data structures such as terms (finitely branching trees) are naturally implemented by second-order recursion: A first-order procedure $f$ passes itself as an argument to a second-order procedure like map, every, foldl, foldr, etc. to recursively apply $f$ to the direct subterms of a term. We present a method for automated termination analysis of such procedures. It extends the approach of argumentbounded functions (i) by inspecting type components and (ii) by adding a facility to take care of second-order recursion. Our method has been implemented and automatically solves the examples considered in the literature. This improves the state of the art of inductive theorem provers, which (without our approach) require user interaction even for termination proofs of simple second-order recursive procedures.
\end{abstract}

\section{Introduction}

Functional programs frequently use higher-order procedures such as map and every that expect functions as parameters [712. For instance, map applies a function to each element of a list and returns the list of the result values. Similarly, every $(p, k)$ yields true iff $p(x)$ evaluates to true for all elements $x$ of a list $k$. If a procedure $f$ calls a higher-order procedure $g$ using $f$ as an argument for $g$, e.g., $g(f, \ldots)$, we say that $f$ is defined by higher-order recursion 814 .

In this paper, we consider the automated termination analysis of functional programs that may use second-order recursion 11 Typical examples arise in algorithms on finitely branching trees such as terms; e.g., applying a substitution to a term or collecting variables in a term. Termination analysis for such programs is non-trivial: In the higher-order theorem provers Isabelle 8 10 14] and PVS [11, the user needs to assist the system to prove termination in these cases. In contrast, the method we propose solves typical termination problems automatically. Furthermore, our method supplies information that allows a theorem prover to generate useful induction axioms for proofs about such programs.

Figure 1 shows an example program. In Fig. 1(a), data types bool, $\mathbb{N}$, and list $[@ A]$ are defined by enumerating the respective data constructors true, false, 0 , succ, $\varnothing$, and "::". Each argument position of a data constructor is assigned a

\footnotetext{
${ }^{1}$ As in 3 , we define the order $o(\tau)$ of base types $\tau$ like $\mathbb{N}$ or list $[\mathbb{N}]$ as 0 ; the order of a functional type $\tau_{1} \times \ldots \times \tau_{n} \rightarrow \tau$ is $1+\max _{i} o\left(\tau_{i}\right)$ for a base type $\tau$.
} 
(a) structure bool $<=$ true, false

structure $\mathbb{N}<=0$, succ $($ pred $: \mathbb{N})$

structure list $[@ A]<=\varnothing,::(h d: @ A, t l: \operatorname{list}[@ A])$

procedure last $(k:$ list $[@ A]): @ A<=$

assume $k \neq \varnothing ;$ if $t l(k)=\varnothing$ then $h d(k)$ else last $(t l(k))$ end

(b) structure variable.symbol $<=$ variable $(\operatorname{var} I D: \mathbb{N})$

structure function.symbol $<=$ func $($ funcID $: \mathbb{N})$

structure term $<=$

$\operatorname{var}($ vsym : variable.symbol),

apply (fsym : function.symbol, args : list $[$ term $])$

procedure every $(p: @ A \rightarrow$ bool, $k:$ list $[@ A]):$ bool $<=$

if $k=\varnothing$ then true else if $p(h d(k))$ then every $(p, t l(k))$ else false end end

procedure groundterm $(t:$ term $)$ : bool $<=$

if ?var $(t)$ then false else every (groundterm, $\operatorname{args}(t))$ end

Fig. 1. A functional program with (a) the first-order procedure last and (b) the secondorder procedure every and second-order recursion in procedure groundterm

selector function; e.g., selector pred denotes the predecessor function. Expressions of the form ? cons $(t)$ check if $t$ denotes a value of the form $\operatorname{cons}(\ldots)$. In Fig. 1(b), procedure every is a second-order procedure that gets a first-order function $p$ as argument. Procedure groundterm uses second-order recursion to check if a term $t$ (modeled by data type term) does not contain any variables.

Our approach extends the method of argument-bounded functions 15,18 that is used, for instance, in the semi-automated verifier VeriFun [17] for termination analysis and the synthesis of suitable induction axioms. Using this approach, termination of every can be easily proved: Selector $t l$ is argument-bounded, which intuitively means $\#(k) \geq \#(t l(k))$ for all lists $k \neq \varnothing$, where $\#(k)$ counts the occurrences of list-constructors $\varnothing$ and :: in $k$ (and thus corresponds to the length of list $k$ plus 1 ). A system-generated difference procedure [15]18, $\Delta_{t l}: \operatorname{list}[@ A] \rightarrow$ bool decides if this inequality is strict for a given list $k$, which is the case if $k \neq \varnothing$. To prove that the second argument of procedure every gets strictly smaller in the recursive call every $(p, t l(k))$, it suffices to show the trivial termination hypothesis $\forall k:$ list $[@ A] . k \neq \varnothing \wedge p(h d(k)) \rightarrow \Delta_{t l}(k)$.

Proving termination of groundterm, however, is challenging and hence is the main problem we tackle in this paper. The key observation is that every applies $p$ only to members $x$ of list $k$. While in Isabelle the user needs to state and prove this knowledge explicitly as a congruence theorem, our approach automatically extracts such information from the definition of every. More specifically, our approach detects that for any instantiation of type variable @ $A$ with a type $\tau$, the number of $\tau$-constructors in each value $x: \tau$ that $p$ is applied to by every is bounded by the number of $\tau$-constructors in the elements $e$ of list $k: \sum_{e \in k} \#(e) \geq$ $\#(x)$. We say that every is call-bounded wrt. $p$. For the second-order recursion in groundterm and $\operatorname{args}(t)=t_{1}:: \ldots:: t_{n}:: \varnothing$ this means $\#\left(t_{1}\right)+\ldots+\#\left(t_{n}\right) \geq$ $\#(x)$. Since $t=\operatorname{apply}(f \operatorname{sym}(t)$, args $(t))$ contains one term-constructor more than 
$\operatorname{args}(t)$, we have $\#(t)>\#\left(t_{1}\right)+\ldots+\#\left(t_{n}\right) \geq \#(x)$, so groundterm is only called recursively with arguments $x$ that are smaller than $t$, which ensures termination.

Formally, we parameterize the size measure \# by a type position so that for $\operatorname{args}(t):$ list $[$ term $]$ we can separately count the list- and term-constructors. This allows us to consider args $:$ term $\rightarrow$ list $[$ term $]$ as argument-bounded wrt. type component term (i. e., $\operatorname{args}(t)$ contains no more term-constructors than $t$ ).

The contributions of this paper are:

(1) An extended notion of argument-boundedness that also considers components of types (Sect. 2), along with a corresponding extension of the estimation calculus to automate size estimation proofs (Sect. 3). These extensions allow our approach to prove termination of several purely first-order procedures that cannot be handled by the original approach in $15[18$.

(2) The novel notion of call-boundedness to automatically prove termination of procedures with second-order recursion (Sect. 4). This extension maintains the advantage that "optimized" induction axioms can be synthesized.

We discuss related work and experimental results in Sect. 5. Proofs of the theorems in this paper are given in [1] and 2] along with further details and examples.

\section{Size Estimation for Polymorphic Data Types}

In this section we define the basic ingredients for size estimation proofs. We begin with a brief account of the programming language $\mathcal{L}$ (which is the input language of $\sqrt{ }$ eriFun [17] and roughly corresponds to the second-order fragment of Haskell with strict evaluation); see [1|2[16] for formal details on $\mathcal{L}$.

\subsection{Programming Language}

The input language $\mathcal{L}$ of $\sqrt{ }$ eriFun consists of definition principles for freely generated polymorphic data types, for first-order and second-order procedures (based on non-mutual recursion 2 case analyses via if-expressions, and functional composition) that operate on these data types, and for statements about the data types and procedures. Each function symbol can be associated with a so-called context requirement, which is stipulated explicitly for procedures (as for last in Fig. (1) and implicitly for all selectors. VeriFun enforces via proof obligations that the context requirement be satisfied for each function call [13] e.g., last, $h d$, and $t l$ may only be called on non-empty lists.

A base type is a type variable @ $A$ or an expression of the form $\operatorname{str}\left[\tau_{1}, \ldots, \tau_{k}\right]$, where $\tau_{1}, \ldots, \tau_{k}$ are base types and $s t r$ is a $k$-ary type constructor $(k \geq 0)$. A type is a base type or an expression of the form $\tau_{1} \times \ldots \times \tau_{k} \rightarrow \tau$ for types $\tau_{1}, \ldots, \tau_{k}, \tau$. Type constructors are defined by expressions of the following form:

$$
\text { structure } \operatorname{str}\left[@ A_{1}, \ldots, @ A_{k}\right]<=\ldots, \operatorname{cons}\left(\operatorname{sel}_{1}: \tau_{1}, \ldots, \operatorname{sel}_{n}: \tau_{n}\right), \ldots
$$

${ }^{2}$ Our approach can be extended to handle mutual recursion without much difficulty. 
The $\tau_{j}$ are base types, and str may only occur as $\operatorname{str}\left[@ A_{1}, \ldots\right.$, @ $\left.A_{k}\right]$ in the $\tau_{j}$. Each cons is called a data constructor and the sel $_{j}$ are called selectors.

We will address type symbols (i.e., type constructors and type variables) in a base type by their position $\pi \in \mathbb{N}^{*}:\left.@ A\right|_{\epsilon}:=@ A,\left.\operatorname{str}\left[\tau_{1}, \ldots, \tau_{k}\right]\right|_{\epsilon}:=s t r$, and $\left.\operatorname{str}\left[\tau_{1}, \ldots, \tau_{k}\right]\right|_{h \pi^{\prime}}:=\left.\tau_{h}\right|_{\pi^{\prime}}$ for $h \in\{1, \ldots, k\} . \operatorname{Pos}(\tau) \subseteq \mathbb{N}^{*}$ denotes the set of all

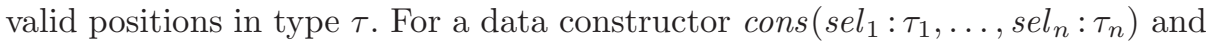
a type symbol $S$, the set

$$
\operatorname{Pos}_{S}(\text { cons }):=\left\{(j, \pi) \in\{1, \ldots, n\} \times \mathbb{N}^{*}\left|\pi \in \operatorname{Pos}\left(\tau_{j}\right), \tau_{j}\right|_{\pi}=S\right\}
$$

contains the positions of all occurrences of $S$ in the selector types of cons, given by a selector number $j$ and a position $\pi$ in $\tau_{j}$. Data constructor cons is called reflexive if Pos $_{\text {str }}$ (cons $) \neq \emptyset$, and irreflexive otherwise.

Subsequently, we let $\Sigma(P)$ denote the signature of all function symbols defined by an $\mathcal{L}$-program $P$. As usual, $\mathcal{T}(\Sigma(P), \mathcal{V})$ denotes the set of all terms over $\Sigma(P)$ and a set $\mathcal{V}$ of variables. We write $\mathcal{T}(\Sigma(P))$ instead of $\mathcal{T}(\Sigma(P), \emptyset)$ for the set of all ground terms over $\Sigma(P) . \Sigma(P)^{c} \subset \Sigma(P)$ contains all data constructors of $P$. $\mathcal{C} \mathcal{L}(\Sigma(P), \mathcal{V})$ is the set of clauses over $\Sigma(P)$, i. e., sets of literals. A literal is an if-free Boolean term or the negation if $(b$, false, true $)$ of such a term.

For a ground typ $3^{3} \tau, \mathbb{V}(P)_{\tau}$ denotes the "values" of type $\tau$ : If $\tau$ is a ground base type, $\mathbb{V}(P)_{\tau}:=\mathcal{T}\left(\Sigma(P)^{c}\right)_{\tau}$, and for each ground type $\tau=\tau_{1} \times \ldots \times \tau_{k} \rightarrow$ $\tau_{k+1}, \mathbb{V}(P)_{\tau}$ contains all closed (i. e., no free variables) $\lambda$-expressions of type $\tau$; e. g., $\lambda t$ : term. groundterm $(t) \in \mathbb{V}(P)_{\text {term } \rightarrow \text { bool }}$.

The call-by-value interpreter eval $_{P}: \mathcal{T}(\Sigma(P)) \mapsto \mathbb{V}(P)$ is a partial function that defines the operational semantics of $\mathcal{L}[2$. The evaluation of a ground term $t$ either (i) succeeds and yields a value $\operatorname{eval}_{P}(t) \in \mathbb{V}(P)$ or (ii) diverges, because a procedure called in $t$ does not terminate.

A procedure procedure $f\left(x_{1}: \tau_{1}, \ldots, x_{n}: \tau_{n}\right): \tau<=$ assume $c_{f} ; B_{f}$ of a program $P$ terminates iff the interpreter eval $_{P}$ returns a value for each procedure call $f\left(q_{1}, \ldots, q_{n}\right)$. The $q_{i}$ are either constructor ground terms or $\lambda$-expressions that contain only calls of arbitrary, but terminating functions. Program $P$ terminates iff all procedures $f$ defined in $P$ terminate.

A universally quantified formula of the form $\forall x_{1}: \tau_{1}, \ldots, x_{n}: \tau_{n}$. $b$, where $b \in \mathcal{T}(\Sigma(P), \mathcal{V})_{b o o l}$, is true iff $P$ terminates and eval $_{P^{\prime}}(b[\vec{q}])=$ true for each terminating program $P^{\prime} \supseteq P$ and all $q_{1}, \ldots, q_{n} \in \mathbb{V}\left(P^{\prime}\right) \unlhd$

\subsection{A Size Measure for Values of Base Types}

Our size measure $\#(t, \pi)$ for terms $t$ is parameterized with a type position $\pi$ so that we can precisely specify which data constructors are to be counted. Figure 2 sketches an implementation of Mergesort: Procedure split splits list $k$ into two lists that are recursively sorted and then merged together by some procedure merge. To prove termination of msort, we need to show that split is strictly argument-bounded: $\#(k, \epsilon)>\#(\operatorname{split}(k), \mathbf{1})$ and $\#(k, \epsilon)>\#(\operatorname{split}(k), \mathbf{2})$. The type position distinguishes between the list-constructors of the pair of lists.

\footnotetext{
${ }^{3}$ A ground (base) type is a (base) type without type variables; e. g., list $[\mathbb{N}]$.

${ }^{4}$ Program $P^{\prime}$ may define additional data types and procedures.
} 


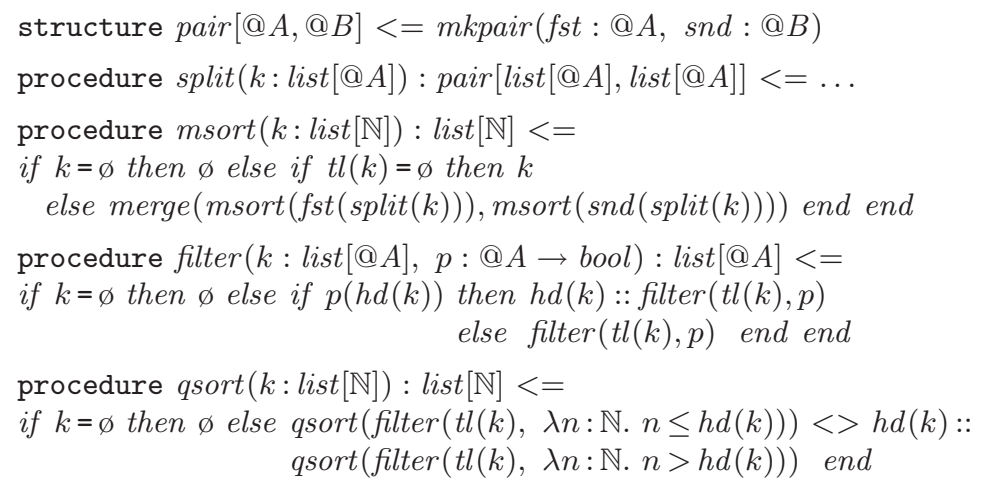

Fig. 2. Implementation of Mergesort (sketch) and Quicksort

Definition 1. For each ground base type $\tau=\operatorname{str}\left[\tau_{1}^{\prime}, \ldots, \tau_{k}^{\prime}\right]$ as in (1) the size measure $\#_{\tau}: \mathcal{T}\left(\Sigma(P)^{c}\right)_{\tau} \times \operatorname{Pos}(\tau) \rightarrow \mathbb{N}$ is defined by $\#_{\tau}\left(\operatorname{cons}\left(t_{1}, \ldots, t_{n}\right), \pi\right):=$

$$
\begin{cases}1 & \text { if } \pi=\epsilon \text { and cons is irreflexive, } \\ 2+\sum_{\left(j, \pi^{\prime}\right) \in \operatorname{Pos}_{s t r}(\text { cons })} \#_{\theta\left(\tau_{j}\right)}\left(t_{j}, \pi^{\prime}\right) & \text { if } \pi=\epsilon \text { and cons is reflexive, } \\ \sum_{\left(j, \pi^{\prime}\right) \in{\operatorname{Pos} @ A_{h}}_{(c o n s)} \#_{\theta\left(\tau_{j}\right)}\left(t_{j}, \pi^{\prime} \pi^{\prime \prime}\right)} & \text { if } \pi=h \pi^{\prime \prime}\end{cases}
$$

where $\theta:=\left\{@ A_{1} / \tau_{1}^{\prime}, \ldots, @ A_{k} / \tau_{k}^{\prime}\right\}$ instantiates the type variables of str. If type $\tau$ is obvious from the context, we will usually omit the type index in $\#_{\tau}$.

Intuitively, the size $\#(t, \pi)$ of a term $t \in \mathcal{T}\left(\Sigma(P)^{c}\right)_{\tau}$ is computed as follows: We replicate the type (and data) constructor definitions so that each type constructor occurs at most once in type $\tau$. Then $\#(t, \pi)$ counts the $\left.\tau\right|_{\pi}$-constructors in $t$. For example, list $[$ list $[\mathbb{N}]]$ is transformed into list $A[$ list $B[\mathbb{N}]]$, so \# $(t, \epsilon)$ counts the list $A$-constructors in $t$ and $\#(t, \mathbf{1})$ counts the list $B$-constructors in $t$.

The formal definition of the size measure above directly uses the type position without needing to replicate any type constructors. Irreflexive data constructors get weight 1 . A reflexive data constructor $\operatorname{cons}\left(\operatorname{sel}_{1}: \tau_{1}, \ldots, \operatorname{sel}_{n}: \tau_{n}\right)$ in a term cons $\left(t_{1}, \ldots, t_{n}\right)$ is counted with weight 5 and we recurse into those $t_{j}: \theta\left(\tau_{j}\right)$ that by definition of cons may also contain str-constructors $\left(\left.\tau_{j}\right|_{\pi^{\prime}}=s t r\right)$. For instance, for $\tau:=\operatorname{list}[$ list $[\mathbb{N}]]$ and $\pi:=\epsilon$ we recursively add the size $\#\left(t_{2}, \epsilon\right)$ of the $t$-component of $t_{1}:: t_{2}$, whereas we do not recurse into the $h d$-component $t_{1}$. Finally, for $\pi=h \pi^{\prime \prime}$ we recursively add up the sizes of those $t_{j}$ that contain $\left.\tau_{h}^{\prime}\right|_{\pi^{\prime \prime}}$ constructors, so we recurse into the occurrences of the $h$-th type parameter @ $A_{h}$ in $\tau_{j}$. For example, for $\tau:=\operatorname{list}[\operatorname{list}[\mathbb{N}]]$, term $t_{1}:: t_{2}$, and $\pi:=\mathbf{1}$, \# list $[\mathbb{N}]\left(t_{1}, \epsilon\right)+$ $\#_{\text {list }[\text { list }[\mathbb{N}]]}\left(t_{2}, \mathbf{1}\right)$ counts the list-constructors of the inner lists.

Example 1. For type $\tau:=\operatorname{list}[\mathbb{N}], \#_{\text {list }[\mathbb{N}]}(t, \epsilon)=2 R+I$ for the numbers $R$ and $I=1$ of occurrences of :: and $\varnothing$ in $t$, respectively, whereas $\#_{\text {list }[\mathbb{N}]}(t, \mathbf{1})$ is the

${ }^{5}$ This simplifies some size estimation proofs; e.g., one can prove that $\operatorname{apply}(f, l)$ is greater than $\operatorname{var}(v)$ without having to check if the argument list $l$ is non-empty. 
sum of the sizes of the elements in list $t$. Note that $\#_{\text {list }[\mathbb{N}]}(\varnothing, \mathbf{1})=0$, whereas $\#_{\text {list }[\mathbb{N}]}(0:: \varnothing, \mathbf{1})=\#_{\mathbb{N}}(0, \epsilon)=1 \neq 0$. Thus $\#_{\text {list }[\mathbb{N}]}(t, \mathbf{1})=0$ iff $t=\varnothing$. This a useful property, cf. the end of Sect. 2.4.

Example 2. For terms $t \in \mathcal{T}\left(\Sigma(P)^{c}\right)_{\text {term }}$, \#term $(t, \epsilon)$ counts the occurrences of term-constructors var (with weight 1) and apply (with weight 2) in $t$.

\subsection{Argument-Bounded Functions}

A function $f$ is called argument-bounded iff the result $f(\ldots, t, \ldots)$ of a function call is bounded by argument $t$ of the call wrt. the size measure (provided that the function may be applied to $t)$; e.g., $\#(t l(k), \epsilon) \leq \#(k, \epsilon)$ for each $k \neq \varnothing$. Such facts are used to show that some parameter $x$ of a procedure $p$ decreases in recursive calls if $f$ is used in the argument of a recursive call; e.g., $p(f(x))$. For the sake of readability we consider only unary functions here, which can be easily generalized to arbitrary arity [1].

Definition 2. A function $f: \tau \rightarrow \tau^{\prime}$ with context requirement $c_{f}$ is $(\pi, \varrho)$ argument-bounded for $\pi \in \operatorname{Pos}(\tau)$ and $\varrho \in \operatorname{Pos}\left(\tau^{\prime}\right)$ iff $(i) \tau$ is a base type with $\left.\tau\right|_{\pi}=\left.\tau^{\prime}\right|_{\varrho}$ and $($ ii $) \#(q, \pi) \geq \#\left(\operatorname{eval}_{P}(f(q)), \varrho\right)$ for all $q \in \mathbb{V}(P)$ with $\operatorname{eval}_{P}\left(c_{f}[q]\right)=$ true 6

Example 3. Procedure last (Fig. 1) is $(\mathbf{1}, \epsilon)$-argument-bounded: The size of the last element of list $k$ is bounded by the sum of the sizes of $k$ 's elements.

Procedure filter (Fig. 2) is $(\epsilon, \epsilon)$-argument-bounded wrt. $k$, because the list of all elements $x$ in $k$ that satisfy $p(x)$ is not longer than $k$.

Selectors are argument-bounded, as they return a component of their input:

Theorem 1. Let $\operatorname{sel}_{j}: \tau \rightarrow \tau_{j}$ be a selector as in (1), $\tau=\operatorname{str}\left[@ A_{1}, \ldots, @ A_{k}\right]$, $\pi \in \operatorname{Pos}(\tau)$, and $\varrho \in \operatorname{Pos}\left(\tau_{j}\right)$. If $\left.\tau\right|_{\pi}=\left.\tau_{j}\right|_{\varrho}$, then $\operatorname{sel}_{j}$ is $(\pi, \varrho)$-argument-bounded.

Example 4. $\operatorname{pred}(\ldots): \mathbb{N} \rightarrow \mathbb{N}$ is $(\epsilon, \epsilon)$-argument-bounded. hd $: \operatorname{list}[@ A] \rightarrow @ A$ is $(\mathbf{1}, \epsilon)$-argument-bounded: The size of the first element of a non-empty list $k$ is bounded by the sum of the sizes of all elements in $k . t l: \operatorname{list}[@ A] \rightarrow \operatorname{list}[@ A]$ is $(\epsilon, \epsilon)$-argument-bounded, as $t l(k)$ contains fewer list-constructors "::" than $k . t l$ is also $(\mathbf{1}, \mathbf{1})$-argument-bounded, because $t l(k)$ contains a subset of the elements in $k$. Finally, selector args $:$ term $\rightarrow$ list $[$ term $]$ is $(\epsilon, \mathbf{1})$-argument-bounded.

\subsection{Difference Procedures}

Using argument-bounded functions, we can establish inequalities like \# $(k, \epsilon) \geq$ $\#(t l(k), \epsilon)$ to ensure that the second argument of procedure every does not increase in the recursive call (cf. Fig. 1). However, this inequality needs to be strict to guarantee termination of every. Strictness of such inequalities is expressed by so-called difference procedures; e.g., $\Delta_{t l}^{\epsilon, \epsilon}: \operatorname{list}[@ A] \rightarrow$ bool returns true iff $\#(k, \epsilon)>\#(t l(k), \epsilon)$.

\footnotetext{
$\overline{6}$ " $q \in \mathbb{V}(P)$ " implicitly means that $\tau$ is instantiated to a ground base type.
} 
(a) procedure $\Delta_{p r e d(\ldots)}^{\epsilon, \epsilon}(x: \mathbb{N}):$ bool $<=$ assume ?succ $(x)$; true procedure $\Delta_{t l}^{\epsilon, \epsilon}(k:$ list $[@ A]):$ bool $<=$ assume ?::(k); true procedure $\Delta_{\text {args }}^{\epsilon, 1}(t:$ term $):$ bool $<=$ assume ?apply $(t)$; true

(b) procedure $\Delta_{h d}^{\mathbf{1}, \epsilon}(k:$ list $[@ A]):$ bool $<=$ assume ?::(k); ?:: $(t l(k))$ procedure $\Delta_{t l}^{\mathbf{1}, \mathbf{1}}(k:$ list $[@ A]):$ bool $<=$ assume ?::(k); true

Fig. 3. Some automatically synthesized difference procedures for selectors

Definition 3. For a $(\pi, \varrho)$-argument-bounded function $f: \tau \rightarrow \tau^{\prime}$ with context requirement $c_{f}, \Delta_{f}^{\pi, \varrho}: \tau \rightarrow$ bool is a $(\pi, \varrho)$-difference function for $f$ iff $(i) \Delta_{f}^{\pi, \varrho}$ also has context requirement $c_{f}$ and (ii) for all $q \in \mathbb{V}(P)$ with $\operatorname{eval}_{P}\left(c_{f}[q]\right)=$ true

$$
\operatorname{eval}_{P}\left(\Delta_{f}^{\pi, \varrho}(q)\right)=\text { true } \Longleftrightarrow \#(q, \pi)>\#\left(\operatorname{eval}_{P}(f(q)), \varrho\right) .
$$

$(\epsilon, \varrho)$-argument-bounded selectors have quite simple difference procedures, because the selector cancels the leading data constructor, cf. Fig. 31(a):

Theorem 2. Let $\mathrm{sel}_{j}: \tau \rightarrow \tau_{j}$ be an $(\epsilon, \varrho)$-argument-bounded selector for some $\varrho$. Then a $(\epsilon, \varrho)$-difference procedure for sel $_{j}$ is given by

$$
\text { procedure } \Delta_{\text {sel }_{j}}^{\epsilon, \varrho}(x: \tau): \text { bool }<=\text { assume ? cons }(x) ; \text { true. }
$$

The synthesis of $(\pi, \varrho)$-difference procedures for selectors with $\pi \neq \epsilon$ is a bit more involved and described in [12]. Figure 3(b) illustrates the idea by examples. $\Delta_{h d}^{\mathbf{1}, \epsilon}$ returns true iff list $k$ contains at least two elements: Since the size of each element in $k$ is $\geq 1$, the size of the first element $h d(k)$ is smaller than the sum of the sizes of all elements in $k$. The uniform synthesis of such procedures uses the fact that $\#(h d(k):: t l(k), \mathbf{1})>\#(h d(k), \epsilon)$ iff $\#(t l(k), \mathbf{1})>0$, i. e., iff ?:: $(t l(k))$.

\section{Estimation Proofs}

So-called estimation proofs can be used to verify that a procedure computes an argument-bounded function. We obtain estimation proofs from the estimation calculus, which is also used to synthesize difference procedures for argumentbounded procedures and to generate termination hypotheses for recursively defined procedures.

\subsection{The Estimation Calculus}

The estimation calculus is used to prove inequalities $\#\left(t_{1}, \pi_{1}\right) \geq \#\left(t_{2}, \pi_{2}\right)$. The inequalities to be shown are given by some set $E$. When proving an inequality, a clause $\Delta$ (called a difference equivalent of $E$ ) is synthesized such that the proved inequality is strict iff one of the literals in $\Delta$ holds.

Definition 4. For a terminating program $P$, let $\Gamma_{\pi, \varrho}$ be a family of $(\pi, \varrho)$ argument-bounded function symbols in $P$. Given a call context $C \in \mathcal{C} \mathcal{L}(\Sigma(P), \mathcal{V})$, the estimation calculus is defined by: 
Language: Estimation tuples of the form $\langle\Delta, E\rangle$, where $\Delta \in \mathcal{C} \mathcal{L}(\Sigma(P), \mathcal{V})$ and $E \subset_{\text {fin }} \mathcal{E}(\Sigma(P), \mathcal{V}):=\left\{\left(t_{1}, \pi_{1}\right) \succcurlyeq\left(t_{2}, \pi_{2}\right) \mid t_{i} \in \mathcal{T}(\Sigma(P), \mathcal{V})_{\tau_{i}}\right.$ for some base types $\tau_{1}, \tau_{2}, \pi_{i} \in \operatorname{Pos}\left(\tau_{i}\right)$ for $i=1,2$ and $\left.\left.\tau_{1}\right|_{\pi_{1}}=\left.\tau_{2}\right|_{\pi_{2}}\right\}$.

Inference Rules: The following estimation rules are given for each type constructor str and data constructors cons, rcons, ircons, ircons ${ }_{1}$, and ircons in $_{2}$ of str, where rcons is reflexive and all ircons $s_{i}$ are irreflexive:7

Identity

$\frac{\langle\Delta, E \uplus\{(t, \pi) \succcurlyeq(t, \pi)\}\rangle}{\langle\Delta, E\rangle}$

Equivalence

(2) $\frac{\left\langle\Delta, E \uplus\left\{\left(t_{1}, \epsilon\right) \succcurlyeq\left(t_{2}, \epsilon\right)\right\}\right\rangle}{\langle\Delta, E\rangle} \quad$ if $C \vdash$ ? ircons $1\left(t_{1}\right)$ and $C \vdash$ ? ircons $_{2}\left(t_{2}\right)$

Strong Estimation

(3) $\frac{\left\langle\Delta, E \uplus\left\{\left(t_{1}, \epsilon\right) \succcurlyeq\left(t_{2}, \epsilon\right)\right\}\right\rangle}{\langle\Delta \cup\{\text { true }\}, E\rangle} \quad$ if $C \vdash$ ?rcons $\left(t_{1}\right)$ and $C \vdash$ ? $\operatorname{ircons}\left(t_{2}\right)$

Strong Embedding

(4)
$\frac{\left\langle\Delta, E \uplus\left\{\left(t_{1}, \epsilon\right) \succcurlyeq\left(t_{2}, \pi_{2}\right)\right\}\right\rangle}{\left\langle\Delta \cup\{\text { true }\}, E \cup\left\{\left(S E L_{j}\left(t_{1}\right), \pi_{1}\right) \succcurlyeq\left(t_{2}, \pi_{2}\right)\right\}\right\rangle}$
if $C \vdash$ ? rcons $\left(t_{1}\right)$ and
$\left(j, \pi_{1}\right) \in$ Pos $_{\text {str }}($ rcons $)$

Argument Estimation

(5) $\frac{\left\langle\Delta, E \uplus\left\{\left(t^{\prime}, \pi^{\prime}\right) \succcurlyeq\left(f\left(t, t_{1}, \ldots, t_{n}\right), \varrho\right)\right\}\right\rangle}{\left\langle\Delta \cup\left\{\Delta_{f}^{\pi, \varrho}\left(t, t_{1}, \ldots, t_{n}\right)\right\}, E \cup\left\{\left(t^{\prime}, \pi^{\prime}\right) \succcurlyeq(t, \pi)\right\}\right\rangle} \quad$ if $f \in \Gamma_{\pi, \varrho}$

Weak Embedding

(6)

$\frac{\left\langle\Delta, E \uplus\left\{\left(t_{1}, \epsilon\right) \succcurlyeq\left(t_{2}, \epsilon\right)\right\}\right\rangle}{\left\langle\Delta, E \cup\left\{\left(S E L_{j}\left(t_{1}\right), \pi\right) \succcurlyeq\left(S E L_{j}\left(t_{2}\right), \pi\right) \mid(j, \pi) \in \text { Pos }_{\text {str }}(\text { rcons })\right\}\right\rangle}$

if $C \vdash$ ? rcons $\left(t_{1}\right)$ and $C \vdash$ ? rcons $\left(t_{2}\right)$

Constructor Wrapping

(7) $\frac{\left\langle\Delta, E \uplus\left\{(t, \varrho) \succcurlyeq\left(\operatorname{cons}\left(t_{1}, \ldots, t_{n}\right), h \pi^{\prime}\right)\right\}\right\rangle}{\left\langle\Delta, E \cup\left\{(t, \varrho) \succcurlyeq\left(t_{j}, \pi \pi^{\prime}\right)\right\}\right\rangle} \quad$ if Pos@ $A_{h}($ cons $)=\{(j, \pi)\}$

Minimum

(8) $\frac{\left\langle\Delta, E \uplus\left\{\left(t_{1}, \epsilon\right) \succcurlyeq\left(t_{2}, \epsilon\right)\right\}\right\rangle}{\left\langle\Delta \cup\left\{? \operatorname{rcons}\left(t_{1}\right) \mid r c o n s \in R\right\}, E\right\rangle}$

if $C \vdash$ ? ircons $\left(t_{2}\right)$ and $R$ is the set of all reflexive constructors of str

Deduction: We write $\left\langle\Delta_{0}, E_{0}\right\rangle \Rightarrow_{\Gamma, C}\left\langle\Delta_{1}, E_{1}\right\rangle$ iff $\left\langle\Delta_{1}, E_{1}\right\rangle$ results from $\left\langle\Delta_{0}, E_{0}\right\rangle$ by applying some estimation rule. $\Rightarrow_{\Gamma, C}^{*}$ denotes the reflexive and transitive closure of $\Rightarrow_{\Gamma, C} \cdot\left\langle\Delta_{0}, E_{0}\right\rangle \Rightarrow_{\Gamma, C}^{*}\left\langle\Delta_{n}, E_{n}\right\rangle$ is called a deduction of $\left\langle\Delta_{n}, E_{n}\right\rangle$ from $\left\langle\Delta_{0}, E_{0}\right\rangle$. We use the notation $\vdash_{\Gamma, C}\left\langle\Delta,\left(t_{1}, \pi_{1}\right) \succcurlyeq\left(t_{2}, \pi_{2}\right)\right\rangle$ iff $\left\langle\emptyset,\left\{\left(t_{1}, \pi_{1}\right) \succcurlyeq\left(t_{2}, \pi_{2}\right)\right\}\right\rangle \Rightarrow_{\Gamma, C}^{*}\langle\Delta, \emptyset\rangle .\left(t_{1}, \pi_{1}\right) \geqslant_{\Gamma, C}\left(t_{2}, \pi_{2}\right)$ denotes the existence of a difference equivalent $\Delta$ with $\vdash_{\Gamma, C}\left\langle\Delta,\left(t_{1}, \pi_{1}\right) \succcurlyeq\left(t_{2}, \pi_{2}\right)\right\rangle$.

$\overline{7}$ The rules are applied from top to bottom. We write $C \vdash$ ? cons $(t)$ iff (i) $t=\operatorname{cons}(\ldots)$ or (ii) ? cons $(t) \in C$ or (iii) $\neg$ ? cons $^{\prime}(t) \in C$ for all str-constructors cons $s^{\prime} \neq$ cons. $S E L_{j}(t)$ stands for $t_{j}$ if $t=\operatorname{cons}\left(\ldots, t_{j}, \ldots\right)$, and abbreviates $\operatorname{sel}_{j}(t)$ otherwise. 
Definition 4 extends the calculus from [1518] by type positions $\pi$ and rule (7) for data constructors such as mkpair (Fig. 2) that just wrap the item of interest; e. g., to show $\#(t, \varrho) \geq \#\left(\operatorname{mkpair}\left(t_{1}, t_{2}\right), \mathbf{1}\right)$ it suffices to show $\#(t, \varrho) \geq \#\left(t_{1}, \epsilon\right)$.

Example 5. We get the following estimation proof for call context $C:=\{k \neq \varnothing\}$ :

$$
\begin{array}{rlrl} 
& \langle\emptyset,\{(k, \epsilon) \succcurlyeq(\text { filter }(t l(k), g), \epsilon)\}\rangle & \\
\Rightarrow_{\Gamma, C}\left\langle\left\{\Delta_{\text {filter }}^{\epsilon, \epsilon}(\operatorname{tl}(k), g)\right\},\{(k, \epsilon) \succcurlyeq(t l(k), \epsilon)\}\right\rangle & \text { by }(5) \\
\Rightarrow_{\Gamma, C}\left\langle\left\{\text { true, } \Delta_{\text {filter }}^{\epsilon, \epsilon}(t l(k), g)\right\},\{(t l(k), \epsilon) \succcurlyeq(t l(k), \epsilon)\}\right\rangle & \text { by (4) } \\
\left.\Rightarrow_{\Gamma, C}\left\langle\left\{\text { true, } \Delta_{\text {filter }}^{\epsilon, \epsilon}(t)(k), g\right)\right\}, \emptyset\right\rangle & \text { by (1) }
\end{array}
$$

In the following, we use expressions of the form (i) $\left(t_{1}, \pi_{1}\right) \geqslant \#\left(t_{2}, \pi_{2}\right)$ and (ii) $\left(t_{1}, \pi_{1}\right)>_{\#}\left(t_{2}, \pi_{2}\right)$ for terms $t_{i} \in \mathcal{T}(\Sigma(P), \mathcal{V})_{\tau_{i}}$ and positions $\pi_{i} \in \operatorname{Pos}\left(\tau_{i}\right)$, $i=1,2$, with $\left.\tau_{1}\right|_{\pi_{1}}=\left.\tau_{2}\right|_{\pi_{2}}$. Such expressions are true iff (i) \# $\left(\operatorname{eval}_{P}\left(t_{1}\right), \pi_{1}\right) \geq$ $\#\left(\operatorname{eval}_{P}\left(t_{2}\right), \pi_{2}\right)$ or (ii) \#( $\left.\operatorname{eval}_{P}\left(t_{1}\right), \pi_{1}\right)>\#\left(\operatorname{eval}_{P}\left(t_{2}\right), \pi_{2}\right)$, respectively.

Theorem 3. The estimation calculus is sound: If $\vdash_{\Gamma, C}\left\langle\Delta,\left(t_{1}, \pi_{1}\right) \succcurlyeq\left(t_{2}, \pi_{2}\right)\right\rangle$, then the following formulas are true (where $x_{1}, \ldots, x_{n}$ are all variables in $C, t_{1}$, and $t_{2}$ such that $x_{i} \in \mathcal{V}_{\tau_{i}}$ for all $\left.i \in\{1, \ldots, n\}\right)$ :

(1) $\forall x_{1}: \tau_{1}, \ldots, x_{n}: \tau_{n} \cdot \bigwedge C \rightarrow\left(t_{1}, \pi_{1}\right) \geqslant \#\left(t_{2}, \pi_{2}\right)$

(2) $\forall x_{1}: \tau_{1}, \ldots, x_{n}: \tau_{n} . \bigwedge C \rightarrow\left[\bigvee \Delta \leftrightarrow\left(t_{1}, \pi_{1}\right)>_{\#}\left(t_{2}, \pi_{2}\right)\right]$

Theorem 4. The set $\left\{\left(t_{1}, \pi_{1}\right) \succcurlyeq\left(t_{2}, \pi_{2}\right) \in \mathcal{E}(\Sigma(P), \mathcal{V}) \mid\left(t_{1}, \pi_{1}\right) \geqslant_{\Gamma, C}\left(t_{2}, \pi_{2}\right)\right\}$ of provable size estimation problems is decidable.

Thus whenever a proof procedure for the estimation calculus finds a proof of $\left(t_{1}, \pi_{1}\right) \geqslant_{\Gamma, C}\left(t_{2}, \pi_{2}\right)$, we know that $t_{1}$ is at least as big as $t_{2}$ by Theorem 3 . If no estimation proof exists, the inequality might still hold, because the estimation calculus is incomplete. However, it is powerful enough to solve termination problems that are relevant in practice, see Sect. 5]

\subsection{Proving Argument-Boundedness of Procedures}

Using the estimation calculus, we can prove argument-boundedness of a procedure $f$ by analyzing the result terms $t_{1}, \ldots, t_{n}$ of $f$ (these are maximal iffree terms outside an if-condition in the body $\left.B_{f}\right)$. The call context $C_{i} \in$ $\mathcal{C} \mathcal{L}(\Sigma(P), \mathcal{V})$ of a result term $t_{i}$ consists of the conditions in $B_{f}$ that lead to $t_{i}$.

Theorem 5. Let procedure $f(x: \tau): \tau^{\prime}<=$ assume $c_{f} ; B_{f}$ be a terminating procedure, $\pi \in \operatorname{Pos}(\tau)$, and $\varrho \in \operatorname{Pos}\left(\tau^{\prime}\right)$ such that $(i) \tau$ is a base type with $\left.\tau\right|_{\pi}=\left.\tau^{\prime}\right|_{\varrho}$ and $(i i) \vdash_{\Gamma, C_{i}}^{f}\left\langle\Delta_{i},(x, \pi) \succcurlyeq\left(t_{i}, \varrho\right)\right\rangle$ for each result term $t_{i}$ of $f$ under call context $C_{i}$ and some $\Delta_{i}$, where $\vdash_{\Gamma, C_{i}}^{f}$ differs from $\vdash_{\Gamma, C_{i}}$ in that the Argument Estimation rule (5) may also be used for each recursive call $f\left(t^{\prime}\right)$ in $t_{i}$.

Then $f$ is $(\pi, \varrho)$-argument-bounded and procedure $\Delta_{f}^{\pi, \varrho}(x: \tau):$ bool $<=B_{\Delta_{f}}$ is a $(\pi, \varrho)$-difference procedure for $f$, where $B_{\Delta_{f}}$ is derived from $B_{f}$ by replacing each result term $t_{i}$ with the disjunction $\bigvee \Delta_{i}$ (represented by if-conditionals). 
procedure $\Delta_{\text {last }}^{\mathbf{1 , \epsilon}}(k:$ list $[@ A]):$ bool $<=$

assume $k \neq \varnothing ;$ if $t l(k)=\varnothing$ then false else true end

procedure $\Delta_{\text {filter }}^{\epsilon, \epsilon}(k:$ list $[@ A], p: @ A \rightarrow$ bool $):$ bool $<=$

if $k=\varnothing$ then false else if $p(h d(k))$ then $\Delta_{\text {filter }}^{\epsilon, \epsilon}(t l(k), p)$ else true end end

Fig. 4. Difference procedures for argument-bounded procedures

Example 6. Procedure last shown in Fig. 1(a) is $(\mathbf{1}, \epsilon)$-argument-bounded, because $\vdash_{\Gamma, C_{1}}^{\text {last }}\left\langle\Delta_{1},(k, \mathbf{1}) \succcurlyeq(h d(k), \epsilon)\right\rangle$ for $C_{1}:=\{k \neq \varnothing, t l(k)=\varnothing\}, \Delta_{1}:=\left\{\Delta_{h d}^{\mathbf{1}, \epsilon}(k)\right\}$,

and $\vdash_{\Gamma, C_{2}}^{\text {last }}\left\langle\Delta_{2},(k, \mathbf{1}) \succcurlyeq(\operatorname{last}(t l(k)), \epsilon)\right\rangle$ for $C_{2}:=\{k \neq \varnothing, \quad t l(k) \neq \varnothing\}, \Delta_{2}:=$ $\left\{\Delta_{t l}^{\mathbf{1}, \mathbf{1}}(k), \Delta_{\text {last }}^{\mathbf{1}, \epsilon}(t l(k))\right\} . \bigvee \Delta_{1}$ simplifies to false and $\bigvee \Delta_{2}$ simplifies to true using the definition of the difference procedures (Fig. 3) and call contexts $C_{1}$ and $C_{2}$. Difference procedure $\Delta_{\text {last }}^{\mathbf{1 , \epsilon}}$ is shown in Fig. 4 The last element of list $k$ is smaller than the sum of the sizes of all list elements if the length of $k$ is $\geq 2 . \diamond$

Example \%. For procedure filter (Fig. 2), the difference procedure $\Delta_{\text {filter }}^{\epsilon, \epsilon}$ wrt. parameter $k$ (Fig. 4) reflects the intuition that the returned sublist of $k$ is shorter than $k$ iff at least one element $x$ of $k$ does not satisfy $p(x)$.

\section{Automated Termination Proofs}

We implicitly assume procedure bodies to be in $\eta$-long form to clearly exhibit indirect function calls; e. g., every $(p, t l(k))$ abbreviates every $(\lambda x: @ A . p(x), t l(k))$ in Fig. 1 because $p={ }_{\eta} \lambda x: @ A . p(x)$. Subterm $p(x)$ is an indirect function call, whereas $p(h d(k))$ and every $(\lambda x: @ A . p(x), t l(k))$ are direct function calls:

Definition 5. A direct call of a function $f$ in a term $t$ is a subterm $f\left(t_{1}, \ldots, t_{n}\right)$ of $t$ that occurs outside a $\lambda$-expression. A subterm $f\left(t_{1}, \ldots, t_{n}\right)$ of $t$ inside a $\lambda$ expression is an indirect call of $f$.

Definition 6. For a procedure or $\lambda$-expression $f$ with body $B_{f}$ and parameters $x_{1}, \ldots, x_{n}$, a procedure or $\lambda$-expression $g$, and $q_{1}, \ldots, q_{n}, q_{1}^{\prime}, \ldots, q_{m}^{\prime} \in \mathbb{V}(P)$, we write $f\left(q_{1}, \ldots, q_{n}\right) \triangleright g\left(q_{1}^{\prime}, \ldots, q_{m}^{\prime}\right)$ iff $B_{f}$ contains a subterm $h\left(t_{1}^{\prime}, \ldots, t_{m}^{\prime}\right)$ under some call context $C$ such that for $\sigma:=\left\{x_{1} / q_{1}, \ldots, x_{n} / q_{n}\right\}, \sigma(h)=_{\eta} g$, $\operatorname{eval}_{P}(\sigma(c))=$ true for all $c \in C$, and $q_{i}^{\prime}=\operatorname{eval}_{P}\left(\sigma\left(t_{i}^{\prime}\right)\right)$ for all $i=1, \ldots, m$.

Intuitively, relation $\triangleright$ means "requires evaluation of". For instance, we have every $($ groundterm, $\operatorname{var}(q):: \varnothing) \triangleright$ groundterm $(\operatorname{var}(q))$.

Now we are ready to state a termination criterion for procedures without second-order recursion. The formulas (ii) of Theorem 6 are so-called termination hypotheses; if these formulas are true, the procedure terminates.

Theorem 6. A procedure procedure $f(x: \tau): \tau^{\prime}<=$ assume $c_{f} ; B_{f}$ terminates if all procedures $g \neq f$ occurring in $B_{f}$ and $c_{f}$ terminate and if there is some $\pi \in \operatorname{Pos}(\tau)$ such that each recursive call $f(t)$ in $B_{f}$ under some call context $C \in$ $\mathcal{C} \mathcal{L}(\Sigma(P), \mathcal{V})$ is a direct procedure call such that $(i) \vdash_{\Gamma, C}\langle\Delta,(x, \pi) \succcurlyeq(t, \pi)\rangle$ for some $\Delta$, and (ii) $\forall x: \tau$. $\bigwedge C \rightarrow \bigvee \Delta$ is true. 
Example 8. Procedure qsort (Fig. 2) terminates. For $\pi:=\epsilon, C:=\{k \neq \varnothing\}$, and any $g,(k, \epsilon) \geqslant_{\Gamma, C}($ filter $(t l(k), g), \epsilon)$ with $\bigvee \Delta=$ true, cf. Example 5 .

Example 9. Procedure termlist.size terminates according to Theorem 6 .

procedure termlist.size $(k: \operatorname{list}[$ term $]): \mathbb{N}<=$

if $k=\varnothing$ then 0

else if ? $\operatorname{var}(h d(k))$

$$
\begin{aligned}
& \text { then } 1+\text { termlist.size }(\operatorname{tl}(k)) \\
& \text { else } 1+\text { termlist.size }(t l(k))+\text { termlist.size }(\operatorname{args}(h d(k)))
\end{aligned}
$$

end end

For position $\pi:=\mathbf{1}$ it is easy to $\operatorname{show}(k, \mathbf{1}) \geqslant_{\Gamma, C_{1}}(t l(k), \mathbf{1})$ and $(k, \mathbf{1}) \geqslant_{\Gamma, C_{2}}$ $(\operatorname{args}(h d(k)), \mathbf{1})$ for the respective call contexts $C_{1}$ and $C_{2}$. The resulting termination hypotheses $\forall k:$ list [term $] . k \neq \varnothing \wedge \ldots \rightarrow \Delta_{t l}^{\mathbf{1}, \mathbf{1}}(k)$ and $\forall k:$ list [term $] . k \neq \varnothing \wedge$ $\neg ? \operatorname{var}(h d(k)) \rightarrow\left(\Delta_{h d}^{\mathbf{1}, \epsilon}(k) \vee \Delta_{\text {args }}^{\epsilon, \mathbf{1}}(h d(k))\right)$ are obviously true, cf. Fig. 3 ,

Example 9 cannot be solved by the original method in 1518, because there a list is always measured by its length (the special case $\pi=\epsilon$ of our theorem).

\subsection{Call-Bounded Procedures}

Call-bounded procedures $f$ are well-behaved in the sense that they call their functional parameter only with arguments of a bounded size: For each sequence $f(g, q) \triangleright^{*} g\left(q^{\prime}\right)$ of procedure calls, the size of $q$ is a bound of the size of $q^{\prime}$. We consider only procedures with two parameters in the following definition for readability reasons; the straightforward generalization is given in [1].

Definition 7. A procedure procedure $f\left(F: \tau^{\prime} \rightarrow \tau^{\prime \prime}, x: \tau\right): \tau^{\prime \prime \prime}<=$ assume $c_{f}$; $B_{f}$ is $(\pi, \varrho)$-call-bounded for $\pi \in \operatorname{Pos}(\tau)$ and $\varrho \in \operatorname{Pos}\left(\tau^{\prime}\right)$ iff $\tau$ is a base type with $\left.\tau\right|_{\pi}=\left.\tau^{\prime}\right|_{\varrho}$ such that $\#(q, \pi) \geq \#\left(q^{\prime}, \varrho\right)$ for all $g \in \mathbb{V}(P)_{\tau^{\prime} \rightarrow \tau^{\prime \prime}}$ and $q \in \mathbb{V}(P)_{\tau}$ with $f(g, q) \triangleright h_{1}(\ldots) \triangleright \ldots \triangleright h_{n}(\ldots) \triangleright g\left(q^{\prime}\right)$, where $h_{i} \neq g$ for all $i=1, \ldots, n$.

Example 10. every is $(\mathbf{1}, \epsilon)$-call-bounded, because parameter $p$ will only be called with an argument $x$ with $\#(k, \mathbf{1}) \geq \#(x, \epsilon)$. More formally, $\#(q, \mathbf{1}) \geq \#\left(q^{\prime}, \epsilon\right)$ whenever $\operatorname{every}(g, q) \triangleright \operatorname{every}\left(g, q_{1}\right) \triangleright \ldots \triangleright \operatorname{every}\left(g, q_{n}\right) \triangleright g\left(q^{\prime}\right)$ for some $n \geq 0$. For the same reason, filter is also $(\mathbf{1}, \epsilon)$-call-bounded.

The next theorem allows us to easily identify many call-bounded procedures.

Theorem 7. A procedure procedure $f\left(F: \tau^{\prime} \rightarrow \tau^{\prime \prime}, x: \tau\right): \tau^{\prime \prime \prime}<=$ assume $c_{f}$; $B_{f}$ is $(\pi, \varrho)$-call-bounded for $\pi \in \operatorname{Pos}(\tau)$ and $\varrho \in \operatorname{Pos}\left(\tau^{\prime}\right)$ if $\tau$ is a base type with $\left.\tau\right|_{\pi}=\left.\tau^{\prime}\right|_{\varrho}$ and $F$ occurs in $B_{f}$ only

(1) in direct function calls $F(t)$ under some call context $C$ such that $\vdash_{\Gamma, C}\langle\Delta,(x, \pi) \succcurlyeq(t, \varrho)\rangle$ for some $\Delta$, or

(2) in direct recursive calls $f\left(F, t^{\prime}\right)$ under some call context $C^{\prime}$ such that $\vdash_{\Gamma, C^{\prime}}\left\langle\Delta^{\prime},(x, \pi) \succcurlyeq\left(t^{\prime}, \pi\right)\right\rangle$ for some $\Delta^{\prime}$.

Example 11. Procedure every (Fig. 1) is easily proved $(\mathbf{1}, \epsilon)$-call-bounded:

(1) $\vdash_{\Gamma, C}\left\langle\left\{\Delta_{h d}^{\mathbf{1}, \epsilon}(k)\right\},(k, \mathbf{1}) \succcurlyeq(h d(k), \epsilon)\right\rangle$, where $C=\{k \neq \varnothing\}$

$(2) \vdash_{\Gamma, C^{\prime}}\left\langle\left\{\Delta_{t l}^{\mathbf{1}, \mathbf{1}}(k)\right\},(k, \mathbf{1}) \succcurlyeq(t l(k), \mathbf{1})\right\rangle$, where $C^{\prime}=\{k \neq \varnothing, p(h d(k))\}$ 
Generalized Detection of Call-Bounded Procedures. Theorem 7 handles the frequently occurring special case of Definition 7 where $h_{1}=\ldots=h_{n}=f$, i. e., the functional parameter $F$ is either called directly or passed to the recursive call $f\left(F, t^{\prime}\right)$ without modification. The theorem can be generalized (i) to allow $f$ to pass $F$ to another call-bounded procedure $h_{i} \neq f$, (ii) to allow modification of $F$ in recursive calls by encapsulating it in a $\lambda$-expression $\lambda x^{\prime} \ldots F\left(t^{\prime \prime}\right) \ldots$, and (iii) to allow $F$ to occur in indirect recursive calls as well, see [1.

\subsection{Proving Termination of Procedures}

The concept of call-bounded procedures allows us to prove termination of procedures that pass themselves to a call-bounded second-order procedure: In the following theorem, the arguments $t$ of direct recursive calls need to decrease, cf. requirements (1) and (2). Indirect recursive calls need to occur via a callbounded procedure $g$, cf. (3). This procedure $g$ must be called with a bounding argument $t^{\prime}$ that is strictly smaller than the argument $x$ of $f$, cf. (4) and (5).

Theorem 8. A procedure $f(x: \tau): \tau^{\prime}<=$ assume $c_{f} ; B_{f}$ terminates if all procedures $g \neq f$ occurring in $B_{f}$ and $c_{f}$ terminate and if there is some $\pi \in \operatorname{Pos}(\tau)$ such that for each direct recursive call $f(t)$ in $B_{f}$ under some call context $C$

(1) $\vdash_{\Gamma, C}\langle\Delta,(x, \pi) \succcurlyeq(t, \pi)\rangle$ for some $\Delta$ and

(2) $\forall x: \tau$. $\bigwedge C \rightarrow \bigvee \Delta$ is true

and for each indirect recursive call $g\left(f, t^{\prime}\right)$ in $B_{f}$ under some call context $C^{\prime}$

(3) procedure $g$ is $\left(\pi^{\prime}, \pi\right)$-call-bounded for some $\pi^{\prime}$,

(4) $\vdash_{\Gamma, C^{\prime}}\left\langle\Delta^{\prime},(x, \pi) \succcurlyeq\left(t^{\prime}, \pi^{\prime}\right)\right\rangle$ for some $\Delta^{\prime}$, and

(5) $\forall x: \tau . \wedge C^{\prime} \rightarrow \bigvee \Delta^{\prime}$ is true.

Example 12. Procedure groundterm of Fig. 1 terminates by Theorem 8 .

(3) every is $(\mathbf{1}, \epsilon)$-call-bounded, see Example 11 (i. e., $\pi:=\epsilon$ )

(4) $\vdash_{\Gamma, C^{\prime}}\left\langle\left\{\Delta_{\text {args }}^{\epsilon, \mathbf{1}}(t)\right\},(t, \epsilon) \succcurlyeq(\operatorname{args}(t), \mathbf{1})\right\rangle$, where $C^{\prime}:=\{\neg$ ?var $(t)\}$

(5) $\forall t:$ term. $\neg$ ? $\operatorname{var}(t) \rightarrow \Delta_{\text {args }}^{\epsilon, 1}(t)$ is trivially true, see Fig. 3

Similarly to [1518, Theorem 8 can be generalized in a straightforward way from a single parameter and type position to a set of parameter indices and positions (e.g., for a lexicographic combination of size orders to prove termination of procedures like the Ackermann function). Furthermore, indirect recursive calls $f\left(t^{\prime \prime}\right)$ may be (deeply) nested within $\lambda$-expressions; e. g., in $g\left(\lambda y . \ldots f\left(t^{\prime \prime}\right) \ldots, t^{\prime}\right)$, see [1] for details and examples.

Induction Axioms. From a terminating procedure one can uniformly synthesize a sound induction axiom. Our method maintains the advantage of the original approach [15,18 that the induction axiom(s) can be optimized by analyzing the termination proof: Some variables can be universally quantified in the induction hypotheses (as in [9]) and irrelevant premises are removed [2]. 


\section{$5 \quad$ Related Work and Experimental Results}

Our method is intended to be used in inductive theorem provers: In this setting it is important that many procedures can be proved terminating without user interaction so that the user can quickly move on to the actual verification task.

In Isabelle 2009 [81014] a termination proof of a procedure with second-order recursion requires the user to state and prove a congruence theorem about the second-order procedure involved. For instance, Isabelle can only prove termination of groundterm when the user has proved and explicitly tagged $k=k^{\prime} \wedge$ $\left(\forall x: @ A . x \in k \rightarrow p(x)=p^{\prime}(x)\right) \rightarrow \operatorname{every}(p, k)=\operatorname{every}\left(p^{\prime}, k^{\prime}\right)$ as a congruence rule about procedure every. Our approach with call-bounded procedures can be considered as automatically discovering and proving congruence theorems such as $k=k^{\prime} \wedge\left(\forall x: @ A\right.$. \# $\left.(x, \epsilon)<\#(k, \mathbf{1}) \rightarrow p(x)=p^{\prime}(x)\right) \rightarrow \operatorname{every}(p, k)=\operatorname{every}\left(p^{\prime}, k^{\prime}\right)$.

In PVS 11] the user needs to supply a measure function that computes the size of a data object, so there is no automation as in our approach.

Since Coq does not offer automated termination analysis either, Barthe et al. 4 suggest an approach that ensures termination by typing. Their system uses sized types, i. e., types that contain information about the size of values. For instance, argument-boundedness of procedure split (cf. Fig. 2) is expressed by assigning type $\operatorname{list}^{\ell}[@ A] \rightarrow \operatorname{pair}\left[\operatorname{list}^{\ell}[@ A], \operatorname{list}^{\ell}[@ A]\right]$ to split, where list $^{\ell}[@ A]$ represents lists of length $\leq \ell$. This analysis is less detailed than ours, because it does not detect the cases when the resulting lists are strictly smaller than the input list. Thus the termination proof of Mergesort fails in this approach, whereas our method succeeds using difference procedures that identify these cases [1. In the terminology of sized types, call-boundedness of every could be expressed by assigning type $\left(@ A^{\ell} \rightarrow\right.$ bool $) \times$ list $\left[@ A^{\ell}\right] \rightarrow$ bool to every, thus constraining the items of the list to be of size $\leq \ell$. The approach by Barthe et al. has not been integrated into Coq.

ACL2 519] offers heuristics for automated termination proofs, but procedures cannot be defined by second-order recursion.

There are also several stand-alone approaches for termination analysis, which are useful if only termination of a procedure is to be proved (i. e., if there is no need to synthesize induction axioms for subsequent proofs about the procedure). As an example, we mention the Haskell termination analyzer by Giesl et al. 6].

Experimental Results. Our approach has been integrated into the verifier $\sqrt{ }$ eriFun, which allows us to compare its performance with Isabelle 8 Table1 1 shows the results of our experiments: We evaluated our approach on 16 representative procedures with second-order recursion, including all examples from [810 1114. These procedures are based on 8 common second-order procedures without second-order recursion (e.g., map, foldl, and every). The set of first-order examples comprises auxiliary procedures for the examples of second-order recursion as

\footnotetext{
${ }^{8}$ See http://www.informatik.tu-darmstadt.de/pm/ aderhold for an experimental version of Verifun and a list of the example procedures.
} 
Table 1. Termination proving capabilities of inductive provers

\begin{tabular}{lcc}
\hline number and category of examples & Isabelle & $\sqrt{\text { eriFun }}$ \\
\hline 16 procedures with second-order recursion & 0 & 15 \\
8 second-order procedures without second-order recursion & 8 & 8 \\
40 first-order procedures without second-order recursion & 24 & 38 \\
\hline 64 procedures in total & 32 & 61 \\
\hline
\end{tabular}

well as the examples from 1518, which we included to make sure that our approach properly subsumes the original approach. Indeed, our approach only fails on procedures that the original approach already fails on (e.g., because a parameter is increased in recursive calls).

Isabelle fails on the examples of second-order recursion, because we only supplied the raw definition of the procedures. When the user states and proves the required congruence theorems, 15 procedures can be shown terminating as well. The remaining procedure is an artificial example (computing the constant zero function) by Krauss [8] that our approach also fails on, because we would need information about the procedure's semantics before proving its termination.

We did not base our method on the sized types approach 4 (although this would be feasible in principle), because the latter cannot solve many naturally occurring examples; e.g., it succeeds on only 21 of 40 first-order procedures in the example set and fails on several common sorting algorithms from [15.

In summary, the experimental results show that the extended estimation calculus (though incomplete) is powerful enough to prove termination of the everyday examples of second-order recursion that frequently occur in practice.

\section{Conclusion}

We extended the concept of argument-bounded functions [1518] in two respects: Firstly, we parameterized the size measure to also consider components of types. This facilitates automated termination proofs (e.g., for the Mergesort implementation in Fig. 2) that were impossible with the original method. Secondly, we identified the new notion of call-boundedness to automate termination proofs for procedures with second-order recursion, which the original method could not cope with at all.

Our method has been integrated into VeriFun [17]. It automatically solves the typical examples of second-order recursion considered in the literature [8]10,11]14] and in this paper within few seconds, whereas other state-of-the-art theorem provers require guidance by the user.

Information gathered from termination analysis is among the most important keys for guiding highly automated verifiers such as ACL2 and VeriFun when selecting useful induction axioms. In all examples of second-order recursion, Verifun synthesizes optimal induction axioms using the results of our method for termination analysis. Although the examples are not overly difficult, such procedures using second-order recursion via map, every, filter, foldl, foldr, 
and similar procedures on other data types (e.g., trees) are widely used in functional programming. Hence our method significantly improves the state of the art in automated theorem proving by reducing the need for user interaction.

Acknowledgment. I am grateful to Jürgen Giesl, Alexander Krauss, Simon Siegler, and Christoph Walther for helpful discussions, to Nathan Wasser for implementing the approach, and to the anonymous referees for valuable feedback.

\section{References}

1. Aderhold, M.: Automated termination analysis for programs with second-order recursion. Technical report, TU Darmstadt (2009)

2. Aderhold, M.: Verification of Second-Order Functional Programs. Doctoral dissertation, TU Darmstadt (2009)

3. Andrews, P.B.: An Introduction to Mathematical Logic and Type Theory: To Truth Through Proof. Kluwer Academic Publishers, Dordrecht (2002)

4. Barthe, G., Grégoire, B., Pastawski, F.: CIC': Type-based termination of recursive definitions in the calculus of inductive constructions. In: Hermann, M., Voronkov, A. (eds.) LPAR 2006. LNCS (LNAI), vol. 4246, pp. 257-271. Springer, Heidelberg (2006)

5. Boyer, R.S., Goldschlag, D.M., Kaufmann, M., Moore, J.S.: Functional instantiation in first-order logic. In: Lifschitz, V. (ed.) Papers in Honor of John McCarthy, pp. 7-26. Academic Press, London (1991)

6. Giesl, J., Swiderski, S., Schneider-Kamp, P., Thiemann, R.: Automated termination analysis for Haskell: From term rewriting to programming languages. In: Pfenning, F. (ed.) RTA 2006. LNCS, vol. 4098, pp. 297-312. Springer, Heidelberg (2006)

7. Peyton Jones, S. (ed.): Haskell 98 Language and Libraries: The Revised Report. Cambridge University Press, Cambridge (2003)

8. Krauss, A.: Automating Recursive Definitions and Termination Proofs in HigherOrder Logic. Doctoral dissertation, TU München, Germany (2009)

9. Manolios, P., Turon, A.: All-termination $(T)$. In: Kowalewski, S., Philippou, A. (eds.) TACAS 2009. LNCS, vol. 5505, pp. 398-412. Springer, Heidelberg (2009)

10. Nipkow, T., Paulson, L.C., Wenzel, M. (eds.): Isabelle/HOL - A Proof Assistant for Higher-Order Logic. LNCS, vol. 2283. Springer, Heidelberg (2002)

11. Owre, S., Shankar, N., Rushby, J.M., Stringer-Calvert, D.W.J.: PVS Language Reference. Computer Science Laboratory, SRI International (November 2001)

12. Paulson, L.C.: ML for the Working Programmer, 2nd edn. Cambridge University Press, Cambridge (1996)

13. Schlosser, A., Walther, C., Gonder, M., Aderhold, M.: Context dependent procedures and computed types in Verifun. In: Proc. of 1st Workshop Programming Languages meet Program Verification. ENTCS, vol. 174, pp. 61-78 (2007)

14. Slind, K.: Reasoning about Terminating Functional Programs. PhD thesis, TU München, Germany (1999)

15. Walther, C.: On proving the termination of algorithms by machine. Artificial Intelligence 71(1), 101-157 (1994)

16. Walther, C., Aderhold, M., Schlosser, A.: The $\mathcal{L} 1.0$ Primer. Technical Report VFR 06/01, Technische Universität Darmstadt (2006)

17. Walther, C., Schweitzer, S.: Verification in the classroom. Journal of Automated Reasoning 32(1), 35-73 (2004)

18. Walther, C., Schweitzer, S.: Automated termination analysis for incompletely defined programs. In: Baader, F., Voronkov, A. (eds.) LPAR 2004. LNCS (LNAI), vol. 3452, pp. 332-346. Springer, Heidelberg (2005) 